\title{
Digital Health
}

\author{
Neville Greening \\ Doctor of Philosophy Academic Mixed Research Methods \\ Osaka University-8-1-1 Aomatani-Higashi, Minoh, \\ OSAKA 562-8558 JAPAN \\ Email: Osaka.university2017@gmail.com \\ DOI: $10.31364 / S C I R J / v 7 . i 7.2019 . P 0719674$ \\ http://dx.doi.org/10.31364/SCIRJ/v7.i7.2019.P0719674
}

\begin{abstract}
The changes that are taking place in the healthcare sector are essential to social and intellectual evolution. Modern medicine is heavily dependent on the written and printed word to store data. However, computers have revolutionized the way information is kept and retrieved in the sense that computers not only cache information, but they also record clinical data and generate medical knowledge.
\end{abstract}

Hence, digital health is the future and is more precise, efficient, experimental, and widely distributed. These features contribute towards technological development that ensures the creation of a new paradigm of medicine. While the adoption of digital health has promised various advantages in the cost savings, elimination of address and the improvement of patients' outcomes, there are still a few challenges that need attention before these advantages can be thoroughly practical. This paper explores the political, social, ethnic, and racial factors that could limit the adaptation of digital health.

Keywords: Health care sector, Intellectual Evolution, Modern Medicine, Revolutionized, Digital Health

\section{INTRODUCTION}

Digital tools in medicine affect how individuals interact with medical care and health care. It has been attributed that digital health has transformed the way people make decisions, exercise and managing their healthcare needs (Elenko, Underwood \& Zohar, 2015). Also, digital health goes beyond the wearable devices to track the fitness goals of the patients or monitor health conditions. Advancement in sensory technology, the wireless capabilities and digital analytics and increase in electronic data are some of the areas that revolutionize digital medicine from the prevention of diseases, the detection of illness and treatment of illness (Topol, 2010). Microsoft and Apple have invested in the areas that offer the customers the capability of tracking, as well as capturing information on their health. Google, another powerhouse in the tech industry, has pushed forward with new medical devices and apps to improve healthcare.

Google, "The rise of mobile and new digital technology, has enabled consumers to take health and wellness into their own hands." (Think with Google, 2017)

The two major areas that have been affected by digital health are wireless technologies and the genomics of common diseases. Importantly, these are the main areas that are characterized by modern innovation and discoveries. The digital devices in medicine promise to revolutionize the future in medicine given the ability to provide distinctive details regarding the biological and physical wellbeing of people (Alpert, 2017). Digital health is said to be the ideal change of clinical medicine, biomedical research, and the commercial sector. Today, consumers and patients have easy access to countless amounts of medical information online. Some health experts encourage their patients to use web messaging and email to tackle these simple medical challenges. While digital health promised the elimination of errors in medicine, few problems still need to be addressed. These include the mistakes of the medicinal electronic charts that do not match with the medications of the patients (Elenko, Underwood \& Zohar, 2015). It clearly shows that there are predicaments that still reverberate through the healthcare system while transitioning to digital health introducing new problems in modern technology.

\section{PROBLEM STATEMENT}

The current usage of healthcare information technology is still low. The commercial websites portray many advertisements, a little amount of disclosure regarding the company, and a significant amount of conflict of interest. Consequently, this has resulted 
in lagging of the digital revolution. It has also resulted in the failure by the leaders in the healthcare industry to accomplish cost savings, increased productivity and improvement in service delivery that is promised by digital health (West \& Miller, 2009).

\section{AIMS OF THE RESEARCH}

This research aims to:

1. To investigate social and political challenges that might limit the application of digital medicine.

2. To explore the impact of racial and ethnic disparities on digital medicine revolution.

\section{LITERATURE REVIEW}

Digital health or E-health is the future of medicine and is indeed indisputable. Undoubtedly, this has had an impact on sensitive healthcare services, for instance, surgery. Fang et al. (2009) investigated the application of digital medicine in hepatic surgery. While the application is still new, so far, the experience has indicated that digital health can be utilized to conduct simulation surgery and to optimize real operations. The results showed that by using digital health, it is possible to minimize the resection volume, as well as reduce the risk in surgical procedures. Hence, digital health can be employed to optimize future surgical operations.

\section{ELIMINATING DISPARITIES}

The abolishment of gaps in the healthcare sector has been one of the leading priorities in the United States of America. The disparities are due to the ethnicity and race of certain individuals. Sadly, this has occurred because of the history of slavery, as well as discrimination in America. Therefore, it has been difficult to eliminate these issues. Also, these issues are not only problems in the healthcare sector but also to other areas in the U.S.A. The population from ethnic backgrounds is afforded to divulge levels of access to healthcare service depending significantly on their race and ethnicity (Alpert, 2017). As a result, inequalities are visible in the mortalities and disease rates across the racial lines in America. Differences are also evident in Infant deaths, Obesity, Diabetes rates, Respiratory and Cardiovascular disease.

\section{METHODOLOGY}

This study will employ a qualitative research design to gather information regarding investigating the factors that could limit the adaptation of digital medicine, including political, social, ethical, and racial issues. Standardized questionnaires will be administered to both health experts and consumers or patients to investigate the attitudes that could hamper the adoption of digital health.

\section{CONCLUSION}

The application of digital health needs to be embraced in the healthcare sector to facilitate the provision of excellent medical services. However, distinct factors, for example, race and ethnicities of individuals are significant challenges that healthcare experiences as they attempt to utilize the trending information technology in the nursing practice. Furthermore, digital health or ehealth is preventive medicine that could save lives and money for the government and private organizations.

Conflicts of Interest: The author declares no conflicts of interest regarding the publication of this paper.

\section{BIBLIOGRAPHY}

1) Alpert, J. S. (2017). Digital Medicine’ Brave New World” 1. The American Journal of Medicine, 130(3), 243-244.

2) Elenko, E., Underwood, L., \& Zohar, D. (2015). Defining digital medicine. Nature Biotechnology, $33(5), 456$.

3) Fang, C. H. et al. (2010). Digital Medicine Technology based on 64-slice Computed Tomography in Hepatic Surgery. Chin Med (Engl), 123(9), 1149-53. 
4) Google thinks with Google. Healthcare, February 2017. https://www.thinkwithgoogle.com/topics/healthcare.html (Accessed, 01/05/2017)

5) Topol, E. J. (2010). Transforming medicine via digital innovation. Science translational medicine, 2(16), 16cm4-16cm4.

6) 6)West, D., \& Miller, E. A. (2009). Digital medicine: Health care in the Internet era. Brookings Institution Press. 